\title{
Fixed Point Theorems for Weak K-Quasi Contractions on a Gene ralized Metric Space with Partial Order
}

\author{
${ }^{1}$ K.P.R. Sastry, ${ }^{2}$ G. Appala Naidu, ${ }^{3}$ Ch. Srinivasa Rao, and ${ }^{4}$ B. Ramu Naidu*
}

\section{ABSTRACT}

In this paper we obtain conditions for a k- quasi contraction on a generalized metric space with a partial order to have a fixed point. Using this, we derive certain known results as corollaries.

Keywords: Generalized metric space, weak k-quasi contraction, fixed point, partial order.

AMS: Subject classification (2010): 54H25, 47H10.

\section{INTRODUCTION}

The concept of metric was introduced by Frechet [10] as an extension of the distance on the real line. Banach contraction principle was given a shape in the context of metric spaces. Later several generalizations of Banach contraction principle were obtained. Also many generalizations of metric spaces were obtained and Banach contraction principle was extended to such spaces. Some of the generalizations of Banach contraction principle were also extended to the generalized versions of metric spaces. Czerwik [8] introduced the concept of b-metric spaces in 1993. Since then fixed point results in b-metric spaces were obtained by several authors. Hitzler and Seda [12] introduced the notion of dislocated metric spaces, in the year 2000. In dislocated metric spaces the self distance of a point may be non-zero and this concept has played a very important role in topology and logical programming. Several authors have studied fixed point theory extensively, [see 2,6,11,14]. Combining several generalizations of metric spaces Jleli and Samet [13] obtained a new generalization in 2015. They termed it as a generalized metric space. Jleli and Samet extended many results available in fixed point theory such as Banach contraction principle, Ciric [6].

Sastry et al.[18] dealt with fixed point results in generalized metric spaces, extended Ciric theorem [7] in generalized metric spaces with less stringent conditions and obtained Banach contraction principle in generalized metric spaces as a corollary. In this paper we obtain fixed point results in generalized metric spaces endowed with a partial order.

\section{PRELIMINARIES}

In this section we give the definition of a generalized metric space, obtain certain properties of generalized metric which we use in the later development. For examples of generalized metric spaces see Sastry et al.[18] We also give various definitions of generalizations of metric spaces which are included in the notion of generalized metric spaces.

\subsection{Definition:-}

A metric on a non- empty set $X$ is a mapping $\quad d: X \times X \rightarrow[0,+\infty)$ satisfying the following conditions:

(2.1.1) for every $(x, y) \in X \times X$,we have

$$
d(x, y)=0 \Leftrightarrow x=y
$$

(2.1.2) for every $(x, y) \in X \times X$,we have

$$
d(x, y)=d(y, x) \text {; }
$$

(2.1.3) for every $(x, y, z) \in X \times X \times X$, we have

$$
d(x, y) \leq d(x, z)+d(z, y) \text {. }
$$

2.2 Definition:- (Czerwik[8]) Let $\mathrm{X}$ be a non-empty set and $d: X \times X \rightarrow[0,+\infty)$ be a given mapping.

We say that $d$ is a $b$-metric on $X$ if it satisfies the following conditions:

(2.2.1) for every $(x, y) \in X \times X$, 


$$
d(x, y)=0 \Leftrightarrow x=y
$$

(2.2.2) for every $(x, y) \in X \times X$,we have

$$
d(x, y)=d(y, x)
$$

(2.2.3) there exists $s \geq 1$ such that ,for every $(x, y, z) \in X \times X \times X$, we have

$$
d(x, y) \leq s[d(x, z)+d(z, y)]
$$

In this case, $(X, d)$ is said to be a $b$-metric space.

The concept of convergence in such spaces in similar to that of metric spaces.

2.3 Definition:-(Hitzler and Seda [12]) Let $\mathrm{X}$ be a non-empty set and $d: X \times X \rightarrow[0,+\infty)$ be a given mapping.

We say that $d$ is a dislocated metric on $X$ if it satisfies the following conditions:

(2.3.1) for every $(x, y) \in X \times X$,

$$
d(x, y)=0 \Rightarrow x=y ;
$$

(2.3.2) for every $(x, y) \in X \times X$,we have

$$
d(x, y)=d(y, x)
$$

(2.3.3) for every $(x, y, z) \in X \times X \times X$,we have

$$
d(x, y) \leq d(x, z)+d(z, y) .
$$

In this case $(X, d)$ is said to be a dislocated metric space.

The motivation of defining this new notion is to get better results in logic programming semantics.

The concept of convergence in such spaces is similar to that of metric spaces.

\subsection{Notation (Jleli and Samet [13]) :-}

Let $X$ be a non-empty set and $D: X \times X \rightarrow[0,+\infty]$ be a given mapping. For every $x \in X$, let us define the set

$$
\mathrm{C}(D, X, x)=\left\{\left\{x_{n}\right\} \subset X \lim _{n \rightarrow \infty} D\left(x_{n}, x\right)=0\right\} .
$$

\subsection{Definition :- (Jleli and Samet [13])}

We say that $D$ is a generalized metric on $X$ if it satisfies the following conditions:

(2.5.1) for every $(x, y) \in X \times X$,

$$
D(x, y)=0 \Rightarrow x=y
$$

(2.5.2) for every $(x, y) \in X \times X$,we have

$$
D(x, y)=D(y, x)
$$

(2.5.3) there exists $\lambda>0$ such that

$$
\text { If }(x, y) \in X \times X,\left\{x_{n}\right\} \in \mathrm{C}(D, X, x) \text {, then } D(x, y) \leq \lambda \limsup _{n \rightarrow \infty} D\left(x_{n}, y\right) .
$$

In this case, we say that the pair $(X, D)$ is a generalized metric space.

We also say that $\lambda$ is a coefficient of $X$. Thus we say that $(X, D)$ is a generalized metric space with coefficient $\lambda$. In general we drop $\lambda$. 
(i) Remark :- Obviously, if the set $\mathrm{C}(D, X, x)$ is empty for every $x \in X$, then $(X, D)$ is a generalized metric space if and only if (2.5.1) and (2.5.2) are satisfied.

(ii) Remark:- It may be observed that metrc spaces, b-metric spaces and dislocated metric spaces are included in the class of generalized metric spaces.

2.6 Definition :- Let $(X, D)$ be a generalized metric space. Let $\left\{x_{n}\right\}$ be a sequence in $X$ and $x \in X$. We say that $\left\{x_{n}\right\} D$-converges to $x$ if

$$
\left\{x_{n}\right\} \in \mathrm{C}(D, X, x) \text {. }
$$

2.7 Proposition :- Let $(X, D)$ be a generalized metric space. Let $\left\{x_{n}\right\}$ be a sequence in $X$ and $(x, y) \in X \times X$. If $\left\{x_{n}\right\} D$-converges to $x$ and $\left\{x_{n}\right\} D$-converges to $y$ then $x=y$.

Proof:- Using the property $(2.5 .3)$, we have

$$
D(x, y) \leq \lambda \lim _{n \rightarrow \infty} \sup D\left(x_{n}, y\right)=0,
$$

which implies from the property $(2.5 .1)$ that $x=y$.

2.8 Definition :- Let $(X, D)$ be a generalized metric space. Let $\left\{x_{n}\right\}$ be a sequence in $X$. We say that $\left\{x_{n}\right\}$ is a $D$-Cauchy sequence if

$$
\lim _{m, n \rightarrow \infty} D\left(x_{n}, x_{n+m}\right)=0 .
$$

2.9 Definition :- Let $(X, D)$ be a generalized metric space. It is said to be $D$-complete if every $D$ - Cauchy sequence in $X$ is convergent to some element in $X$.

2.10 Definition :- Let $f: X \rightarrow X$ be a self map and $x \in X$. Write $f^{1}(x)=f(x)$ and $f^{n+1}(x)=f\left(f^{n}(x)\right)$ for $n=1,2,3, \ldots$ Then $\left\{f^{n}(x)\right\}$ is called the sequence of iterates of $f$ at $x$.

Jle li and Samet [13] extended Banach contraction principle to generalized metric spaces as follows.

2.11 Theorem :- ( Jleli and Samet [1] ,Proposition 3.2)(Banach contraction principle for generalized metric spaces).

Let $(X, D)$ be a complete generalized metric space and $f: X \rightarrow X$

be such that $D(f(x), f(y)) \leq k D(x, y)$ for some $k \in[0,1)$ and for all $x, y \in X$.

Suppose there exists $x_{0} \in X$ such that $\alpha=\sup _{\mathrm{n}} D\left(x_{0}, f^{n}\left(x_{0}\right)\right)<\infty$ Then $\left\{f^{n}\left(x_{0}\right)\right\}$ converges to some w $\in X$ and $\mathrm{w}$ is a fixed point of $f$. Further if $\mathrm{w}^{\prime}$ is another fixed point of $f$ with $\mathrm{D}\left(\mathrm{w}, \mathrm{w}^{\prime}\right)<\infty$ then $\mathrm{w}^{\prime}=\mathrm{w}$.

2.12 Theorem :- Let $(X, D)$ be a generalized metric space. Suppose $\left\{x_{n}\right\} \subseteq X, x \in X$ and $x_{n} \rightarrow X$. Then $D(x, x)=0$

Proof:- We have $D(x, x) \leq \lambda \limsup _{n \rightarrow \infty} D\left(x_{n}, x\right)=\lambda \lim _{n \rightarrow \infty} D\left(x_{n}, x\right)=\lambda .0 \quad$ (since $x_{n} \rightarrow \mathrm{x}$ )

$$
n \rightarrow \infty \quad n \rightarrow \infty \quad=0
$$

Hence $D(x, x)=0$. 
2.13 Theorem :- Let $(X, D)$ be a generalized metric space and $x \in X$.

Suppose $\mathrm{C}(D, X, \lambda) \neq \phi$. Then $D(x, x)=0$.

Proof:- $C(D, X, x) \neq \phi \Rightarrow \exists\left\{x_{n}\right\} \in \mathrm{C}(D, X, x) \Rightarrow x_{n} \rightarrow x \Rightarrow D(x, x)=0$.

(byTheorem 2.12)

\section{MAIN RESULTS}

In this section we define a generalized metric space with partial order, weak $k$-quasi contraction and obtain conditions for a weak $k$-quasi contractive self map on a generalized metric space with a partial order to have a fixed point.

3.1 Definition :- $L$ et $(X, D)$ be a generalized metric space and $\leq$ be a partial order on $X$. Then we say that $(X, D, \leq)$ is a generalized metric with a partial order. If $x, y \in X$ and either $x \leq y$ or $y \leq x$ then we say that $x$ and $y$ are comparable.

If $\left\{x_{n}\right\}$ is a sequence in $X$ such that $x_{n} \leq x_{n+1} \forall n$, then we say that $\left\{x_{n}\right\}$ is an increasing sequence;

If $x_{n+1} \geq x_{n} \forall n$, we say that $\left\{x_{n}\right\}$ is a decreasing sequence.

Suppose $(X, D, \leq)$ is a generalized metric space with a partial order and $\quad f: X \rightarrow X$. We say that $f$ is an increasing function if $x \leq y \Rightarrow f(x) \leq f(y)$;

we say that $f$ is decreasing if $x \geq y \Rightarrow f(x) \leq f(y)$.

3.2 Definition:- (Jleli and Samet [13]) Suppose $(X, D, \leq)$ is a generalized metric space with a partial order. We say that

(3.2.1) $X$ is D-regular (increasing) if $\left\{x_{n}\right\}$ is an increasing sequence in $X,\left\{x_{n}\right\}$ is D-convergent to $X$ implies $x_{n} \leq x \forall n$

and $x_{n} \leq y \forall n$ implies $x \leq y$.

(3.2.2) $X$ is D-regular (decreasing) if $\left\{x_{n}\right\}$ is a decreasing sequence in $X,\left\{x_{n}\right\}$ is D-convergent to $x=>x_{n} \geq x \forall n \quad$ and $x_{n} \geq y \forall n$ implies $x \geq y$.

3.3 Definition :- (Jleli and Samet [13]) Suppose $(X, D, \leq)$ is a generalized metric space with partial order,

$f: X \rightarrow X$ and $k \in(0,1)$. We say that fis a weak $k$-contraction if

$D(f(x), f(y)) \leq k D(x, y)$ whenever $x$ and $y$ are comparable.

We say that $f$ is R-type contraction (Vats [19]) if $\exists k$ and $q$ such that $k \in(0,1)$ and $q \geq 1+2 k$ such that

$D(f x, f y) \leq k \max \left\{\frac{1}{2} D(x, y), D(x, f x), D(y, f y), \frac{D(x, f y)+D(y, f x)}{q}\right\}$ for $x \neq y$, whenever

$x$ and $y$ are comparable.

We say that fis a weak $k$-quasi contraction if

whenever $x$ and $y$ are comparable.

We say that fis weak continuous (increasing) if $\left\{x_{n}\right\}$ is an increasing sequence in $X$,

and $\left\{x_{n}\right\}$ is D-convergent to $x=>\left\{f\left(x_{n}\right)\right\} D$-converges to $f(x)$. 
We say that $f$ is weak continuous (decreasing) if $\left\{x_{n}\right\}$ is a decreasing

sequence in $X,\left\{x_{n}\right\}$ D-converges to $x=>\left\{f\left(x_{n}\right)\right\}$ D-converges $f(x)$.

3.4 The orem :- Suppose $(X, D, \leq)$ is a D-complete generalized metric space with a partial order .

Suppose the following conditions hold in $X$.

$$
\text { (3.4.1) } \quad(X, D, \leq) \text { is D-regular (increasing). }
$$

(3.4.2) $\quad f: X \rightarrow X$ is an increasing function.

(3.4.3) fis a weak $k$-quasi contraction for some $k \in(0,1)$.

(3.4.4) There exists $x_{0} \in X$ such that $x_{0} \leq f\left(x_{0}\right)$ and

$$
\begin{aligned}
& \alpha=\sup _{n} D\left(x_{0}, f^{n}\left(x_{0}\right)\right)<\infty \\
& \text { and } D\left(f^{n}\left(x_{0}\right), f^{n+1}\left(x_{0}\right)\right) \leq k^{n} \alpha \forall n
\end{aligned}
$$

Then $\left\{f^{n}\left(x_{0}\right)\right\}$ D-converges to some $w \in X$.

If $\lim \sup _{\mathrm{n}} D\left(f^{n}\left(x_{0}\right), f(w)\right)<\infty$ and $k \lambda<1$ then $w$ is a fixed point off. $\infty$ then $w=w$,

More over if $w^{\prime}$ is another fixed point of f comparable with $w$ such that $D\left(w, w^{\prime}\right)<\infty$ and $D\left(w^{\prime}, w^{\prime}\right)<$

Proof: $-x_{0} \leq f\left(x_{0}\right)=>f\left(x_{0}\right) \leq f^{2}\left(x_{0}\right)$ (since $f$ is increasing)

$$
=>f^{2}\left(x_{0}\right) \leq f^{3}\left(x_{0}\right)
$$

In general $f^{n}\left(x_{0}\right) \leq f^{n+1}\left(x_{0}\right)$ for $n=0,1,2, \ldots \ldots \ldots$

Thus $\left\{f^{n}\left(x_{0}\right)\right\}$ is an increasing sequence.

We first show that $D\left(f^{n}\left(x_{0}\right), f^{n+m}\left(x_{0}\right)\right) \leq k^{n} \alpha$ for $n=0,1,2, \ldots \ldots \ldots$

The result is true if $n=0$, by (3.4.4.1)

$$
\text { and } m=0,1,2, \ldots \ldots
$$

Now assume the truth for $n$, i.e. $D\left(f^{n}\left(x_{0}\right), f^{n+m}\left(x_{0}\right)\right) \leq k^{n} \alpha$

$$
\text { for } m=0,1,2, \ldots
$$

We show that $D\left(f^{n+1}\left(x_{0}\right), f^{n+1+m}\left(x_{0}\right)\right) \leq k^{n+1} \alpha$ for $m=0,1,2, \ldots$

From (3.4.5) we get $f^{n}\left(x_{0}\right)$ and $f^{n+m}\left(x_{0}\right)$ are comparable; infact

$$
f^{n}\left(x_{0}\right) \leq f^{n+m}\left(x_{0}\right)
$$

Hence $\mathrm{D}\left(f^{n+1}\left(x_{0}\right), f^{n+1}\left(x_{0}\right)\right) \leq k \max \left\{D\left(f^{n}\left(x_{0}\right), f^{n}\left(x_{0}\right)\right), D\left(f^{n}\left(x_{0}\right), f^{n+1}\left(x_{0}\right)\right)\right.$

$$
\begin{aligned}
D\left(f^{n}\left(x_{0}, f^{n+1}\left(x_{0}\right)\right), D\right. & \left.\left(f^{n}\left(x_{0}\right), f^{n+1}\left(x_{0}\right)\right), D\left(f^{n}\left(x_{0}\right), f^{n+1}\left(x_{0}\right)\right)\right\} \\
& \leq k \cdot k^{n} \alpha(b y(3.4 .6)) \\
& =k^{n+1} \alpha .
\end{aligned}
$$

From this it follows that (3.4.7) is true if $m=0$.

Assume the truth of (3.4.7) for $m$.

$$
\begin{aligned}
& \text { Now } D\left(f^{n+1}\left(x_{0}\right), f^{n+1+m+1}\left(x_{0}\right) \leq k \max \left\{D \left(f^{n}\left(x_{0}\right), f^{n+1+m}\left(x_{0}\right), D\left(f^{n}\left(x_{0}\right), f^{n+1}\left(x_{0}\right)\right),\right.\right.\right. \\
& \qquad \begin{array}{l}
\left.D\left(f^{n+1+m}\left(x_{0}\right), f^{n+1+m+1}\left(x_{0}\right)\right), D\left(f^{n}\left(x_{0}\right), f^{n+1+m+1}\left(x_{0}\right)\right), D\left(f^{n+1+m}\left(x_{0}\right), f^{n+1}\left(x_{0}\right)\right)\right\} \\
\left(\operatorname{since} f^{n}\left(x_{0}\right), f^{n+1+m}\left(x_{0}\right) \text { are comparable, by }(3.4 .8)\right) \\
\leq k \max \left\{k^{n} \alpha, k^{n} \alpha, k^{n+1+m} \alpha, k^{n} \alpha, k^{n+1+m} \alpha\right\} \\
\quad=k \cdot k^{n} \alpha \\
=k^{n+1} \alpha .
\end{array}
\end{aligned}
$$

Hence (3.4.7)

holds for $m=0,1,2, \ldots \ldots \ldots$.

Consequently (3.4.6) holds. Hence 
$D\left(f^{n}\left(x_{0}\right), f^{n+m}\left(x_{0}\right)\right) \leq k^{n} \alpha$ for $n=0,1,2, \ldots \ldots$ and $m=0,1,2, \ldots \ldots$.

This shows that $\left\{f^{n}\left(x_{0}\right)\right\}$ is a Cauchy seguence and hence

converges to a limit, say, $w \in X$.

Since $(X, D, \leq)$ is D-regular (increasing) it follows that $f^{n}\left(x_{0}\right) \leq w$ for every $n$.

Now $D\left(f^{n+1}\left(x_{0}\right), f(w)\right) \leq k \max \left\{D\left(f^{n}\left(x_{0}\right), w\right), D\left(f^{n}\left(x_{0}\right), f^{n+1}\left(x_{0}\right)\right), D(w, f(w)), D\left(f^{n}\left(x_{0}\right), f(w)\right), D\left(w, f^{n+1}\left(x_{0}\right)\right)\right\}$.

Let $\in>0$. Then there exists $N$ such that $D\left(f^{n}\left(x_{0}\right), w\right)<\in$ for $n \geq N$.

Hence for $n \geq N$ we have $D\left(f^{n+1}\left(x_{0}\right), f(w)\right) \leq k \max \left\{\in, k^{n} \alpha, \lambda \limsup _{n} D\left(f^{n}\left(x_{0}\right), f(w)\right)\right.$,

Suppose $k \lambda<1$

$$
\left.\limsup _{n} D\left(f^{n}\left(x_{n}\right), f(w), \in\right)\right\} \text {---- (3.4.9) }
$$

Then $\limsup _{n} D\left(f^{n+1}\left(x_{0}\right), f(w)\right) \leq \max \left\{k \in, 0, k \lambda \limsup _{n} D\left(f^{n}\left(x_{0}\right), f(w)\right)\right.$,

$\left.k \limsup _{n} D\left(f^{n}\left(x_{0}\right), f(w)\right), k \in\right\} \quad($ from (3.4.9))

$\leq \max \left\{\max _{\{}\{(k, k \lambda)\}\right.$. limsup $_{n} D\left(f^{n}\left(x_{0}\right), f(w), k \in\right\}$

(since $k \lambda<1$ and $k<1$ )

Therefore $\lim \sup _{n} D\left(f^{n}\left(x_{0}\right), f(w)\right)=0$, since $\in$ is arbitrary and

lim $\sup _{n} D\left(f^{n}\left(x_{0}\right), f(w)\right)<\infty$ (by hypothesis)

$$
\begin{aligned}
& \therefore f^{n}\left(x_{0}\right) \rightarrow f(w) . \\
& \therefore f(w)=w
\end{aligned}
$$

Therefore $w$ is a fixed point of $f$.

Suppose w'is also a fixed point off comparable with $\mathrm{w}$ such that $D\left(w, w^{\prime}\right)<\infty$ and $\quad D\left(w^{\prime}, w^{\prime}\right)<\infty$. Then $D\left(w, w^{\prime}\right)=D\left(f(w), f\left(w^{\prime}\right)\right) \leq k \max$

$\left\{D\left(w, w^{\prime}\right), D(w, f(w)), D\left(w^{\prime}, f\left(w^{\prime}\right), D\left(w, f\left(w^{\prime}\right), D\left(w^{\prime}, f(w)\right)\right\}\right.\right.$

$$
\begin{aligned}
& =k \max \left\{D\left(w, w^{\prime}\right), D(w, w), D\left(w^{\prime}, w^{\prime}\right), D\left(w, w^{\prime}\right), D\left(w^{\prime}, w\right)\right\} \\
& =k \max \left\{D\left(w, w^{\prime}\right), 0, D\left(w^{\prime}, w^{\prime}\right)\right\} \\
& \left.=k \max \left\{D\left(w, w^{\prime}\right), 0,0\right\} \text { (since } w^{\prime} \text { is a fixed point with } D\left(w, w^{\prime}\right)<\infty\right) \\
& =k D\left(w, w^{\prime}\right)
\end{aligned}
$$

Therefore $D\left(w, w^{\prime}\right)=0\left(\right.$ since $\left.D\left(w, w^{\prime}\right)<\infty\right)$

Therefore $w=w^{\prime}$.

Note:- If we replace (3.4.1) by (3.4.1) $)^{1} f: X \rightarrow X$ is weak continuous (increasing) then also the conclusion of theorem 3.4 holds. However, (3.4.1) is a condition on the space $X$ while $(3.4 .1)^{1}$ is a condition of the map $f$.

The following Theorem can be proved following the lines of proof of the above theorem.

3.5 Theorem:- Suppose $(X, D, \leq)$ is a D-complete generalized metric space with a partial order.

Suppose the following conditions hold in $X$.

(3.5.1) $(X, D, \leq)$ is D-regular(decreasing).

(3.5.2) $f: X \rightarrow X$ is a decreasing function.

(3.5.3) $f$ is a weak $k$-quasi contraction for some $k \in(0,1)$

(3.5.4) there exists $x_{0} \in X$ such that $x_{0} \geq f\left(x_{0}\right)$ and

$\alpha=\sup _{n} D\left(x_{0}, f^{n}\left(x_{0}\right)\right)<\infty$ and $D\left(f^{n}\left(x_{0}\right), f^{n+1}\left(x_{0}\right)\right) \leq k^{n} \alpha$ for all $n$.

Then $\left\{f^{n}\left(x_{0}\right)\right\} D$-converges to some $w \in X$.

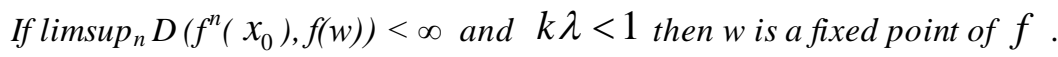

Moreover if $w^{\prime}$ is also a fixed point off comparable with $w$ such that $D\left(w, w^{\prime}\right)<\infty$ and $D\left(w^{\prime}, w^{\prime}\right)<\infty$ then $w=w^{\prime}$.

Note:- If we replace (3.5.1) by (3.5.1)' $f: X \rightarrow X$ is weak continuous (decreasing), still the conclusion of the theorem holds. 
3.6 Corollary :- (Vats et al.[19],Theorem 0.1) Let $(X, D, \leq)$ be a generalized metric space with a partial order and $(X, D, \leq)$ be D-regular (increasing).

Let $f: X \rightarrow X$ satisfy the following.

$f$ is R-type contraction. (i.e)

$D(f x, f y) \leq k \max \left\{\frac{1}{2} D(x, y), D(x, f x), D(y, f y), \frac{D(x, f y)+D(y, f x)}{q}\right\}$ for $x \neq y$, whenever

$x$ and $y$ are comparable.

where $\mathrm{k}$ and $\mathrm{q}$ are reals such that $k \in(0,1)$ and $q \geq 1+2 k$

$f$ is an increasing function, $q \geq 2$ and $k \lambda<1$

Let us assume that there exists a point $x_{0} \in X$ such that $x_{0} \leq f\left(x_{0}\right)$

$$
\alpha=\sup _{n} D\left(x_{0}, f^{n}\left(x_{0}\right)\right)<\infty
$$

and

$$
D\left(f^{n}\left(x_{0}\right), f^{n+1}\left(x_{0}\right)\right) \leq k^{n} \alpha \forall n
$$

Then the sequence $\left\{f^{n}\left(x_{0}\right)\right\}$ converges to some $z \in X$. If $D\left(f(z), x_{0}\right)<\infty$ and

$$
D(z, f(z))<\infty \text {, then } \mathrm{z} \text { is a fixed point of } f \text {. }
$$

Proof :- Under the given conditions it follows that $f$ is a k- quasi contraction and hence the conclusion follows from Theorem 3.4

Note:- Here also the condition: $(X, D, \leq)$ is D-regular (increasing) can be replaced by the condition: $f: X \rightarrow X$ is weak continuous (increasing)

\section{ACKNOWLEDGEMENT}

Fourth Author B.Ramu Naidu is grateful to Special officer, AU.P.G.Centre, Vizianagaram for providing necessary permissions and facilities to carry on this research.

\section{REFERENCES}

[1]. Aage, CT,Salunke, JN:The results on fixed points in dislocated and dislocated quasi-metric

space.Appl.Math.Sci.2(59),2941-2948 (2008)

[2]. Ahamad,MA,Zeyada,FM,Hasan,GF.Fixed point theorems in generalized types of dislocated metric spaces and its applications.Thai J.Math.11,67-73 (2013)

[3]. Akkouchi, M:Common fixed point theorems for two self mappings of a bmetric space under an implicit relation. Hacet. J.Math.Stat.40(6),805-810 (2011)

[4]. Berinde, V:Sequences of operators and fixed points in quasi metric spaces. Stud.Univ. Babes-Bolyai, Math. 41,23-27 (1996)
[5]. Boriceanu, M, Bota,M,Petrusel, A:Multivalued fractals in b-metric spaces. Cent.Eur.J.Math. 8(2),367-377 (2010)

[6]. Ciric,LB:A generalization of Banach's contraction principle. Proc. Am.Math,Soc.45(2), 267-273 (1974)

[7]. L.B.Ciric, A remark on Rhoades fixed point theorem for non-self mappings, Internat . J. Math.\& Math.Sci., 16(2) (1993), 397- 400.

[8]. Czerwik, S: Contraction mappings in bmetric spaces. Acia Math. Inform. Univ.Ostrav. 1,5-11 (1993)

[9]. Czerwik,S,Dlutek,K,Sing,SL:Round-off stability of iteration procedures for setvalued operators in b-metric spaces.J.Natur.Phys.Sci.11,87-94 (2007)

[10]. Frechet: Sur quelques points duo calcul fonctionel, Rendiconti del Circolo Mahematicodi. Palermo. 22: 1-74, 1906.

[11]. Hitzler, P:Generalized metrics and topology in logic programming semantics. Dissertation,Faculty of Science, National University of Ireland, University College,Cork (2000) 
[12]. Hitzler ,P and Seda, AK:Dislocated topologies. J.Electr.Eng.51(2),3-7 (2000)

[13]. M. Jleli and B. Samet, A generalized metric space and related fixed point Theorems, Fixed point Theory and Applications, (2015), 2012:61.DOI: $10.1186 / \mathrm{s} 13663-015-0312-$ 7.

[14]. Karapinar,E,Salimi,P:Dis located metric space to metric spaces with some fixed point theorems.Fixed Point Theory Appl.2013, 222 (2013)

[15]. Kirk, W, Shahzad, N: b-Metric spaces. Fixed Point Theory in Distance Spaces, PP 113-131.Springer,Berlin(2014)

[16]. Popovic,B,Radenovic, S and Shukla,S:Fixed point results to tvs cone b-metric spaces.Gulf J.Math. 1,5164(2013)

[17]. B.E.Rhoades, A fixed point theorem for some non-self mappings, Math.Japonica. 23(4) (1978),457-459.

[18]. Sastry,K.P.R., Appalanaidu,G., Srinivasa Rao, ch., and Ramunaidu,B: Fixed point theorems for weak k-quasi contractions on a generalized metric space, paper presented at RADMAS- 2016 held at Vis akhapatnam during Nov.17-18, 2016.

[19]. VATS, R.K; MANJU GREWAL and AMIT KUMAR; Some fixed point theorems in generalized metric spaces, Adv: Fixed point Theory, 6(2016), No.3,254-261, ISSN: 1927-6303 